\title{
Histologic coagulative tumour necrosis as a prognostic indicator of aggressiveness in renal, lung, thyroid and colorectal carcinomas: A brief review
}

\author{
ROSARIO CARUSO ${ }^{1}$, ANTONINO PARISI ${ }^{2}$, ANNA BONANNO ${ }^{1}$, DOMENICA PAPARO ${ }^{2}$, \\ EMILIA QUATTROCCHI ${ }^{1}$, GIOVANNI BRANCA ${ }^{1}$, MARCO SCARDIGNO $^{1}$ and FRANCESCO FEDELE ${ }^{1}$ \\ Departments of ${ }^{1}$ Human Pathology and ${ }^{2}$ Surgical Sciences, University of Messina, Messina, Italy
}

Received May 18, 2011; Accepted August 30, 2011

DOI: $10.3892 / \mathrm{ol} .2011 .420$

\begin{abstract}
Tumour growth involves two essential deviations from the normal state including the induction of proliferative stimuli, and simultaneous suppression of potentially compensatory cell death. It has been suggested that the development of invasive cancer involves a progressive switch from predominantly apoptotic to necrotic tumour cell death. The presence of tumour necrosis in pathologic specimens may not only reflect tumour biology, but also provide additional beneficial prognostic information. This review emphasises the role of tumour necrosis as an additional prognostic factor for patients with certain types of epithelial neoplasms.
\end{abstract}

\section{Contents}

1. Introduction

2. Tumour necrosis in renal cell carcinoma

3. Tumour necrosis in lung carcinoma

4. Tumour necrosis in thyroid carcinoma

5. Tumour necrosis in colorectal cancer

6. Conclusion

\section{Introduction}

The presence of tumour necrosis in the pathological specimen may reflect tumour biology and provide additional beneficial prognostic information. It is believed to occur when tumours outgrow their blood supply and, therefore, histologic tumour necrosis has been proposed as an indicator of tumour aggressiveness that generally leads to poor clinical outcomes. Previous studies investigated the utility of tumour necrosis

Correspondence to: Professor Rosario Caruso, Department of Human Pathology, University of Messina, Messina I-98125, Italy

E-mail: rosariocaruso@tin.it

Key words: tumour necrosis, histopathology, prognosis as a prognostic factor for patients with tumours and reported conflicting results $(4,15)$. Given this controversy, the present study reviews main morphological aspects of tumour necrosis and its role as a predictor of clinical prognosis for patients with certain types of epithelial neoplasm (renal, lung, thyroid and colorectal carcinoma), where tissue necrosis has been extensively investigated.

\section{Tumour necrosis in renal cell carcinoma}

Currently tumour stage, size, renal cell carcinoma subtype, and nuclear grade are widely accepted as significant pathologic prognostic indicators for renal cell carcinoma (12). However, the findings from the study of Sengupta et al (15) underscore the importance of histologic coagulative tumour necrosis as a predictor of aggressive forms of renal cell carcinoma. Necrosis was found in $914(30 \%)$ of the 3009 renal cell tumours analysed, but the prevalence of this feature differed significantly by histological subtype (15). Tumour necrosis was observed in $196(47 \%)$ patients with papillary renal cell carcinoma and in $28(20 \%)$ patients with chromophobe renal cell carcinoma, compared with $690(28 \%)$ patients with clear cell renal carcinomas (15). The associated viable tumour adjacent to areas of necrosis was typically high-grade (Grade 3 or 4) renal cell carcinomas. The presence of coagulative tumour necrosis had relatively different prognostic implications for the three subtypes. For patients with clear cell and chromophobe renal cell carcinoma, 10-year cancer-specific survival was 77.6 and $90.0 \%$, respectively, in the absence of tumour necrosis, but only 29.2 and $68.3 \%$, respectively, in its presence. In contrast, despite a higher prevalence of coagulative tumour necrosis in papillary renal cell carcinoma, absence of tumour necrosis was not of prognostic significance. Moreover, the association between coagulative tumour necrosis and mortality from clear cell carcinoma was observed on multivariate analysis after adjusting for tumour size, TNM stage, and nuclear grade (15). Thus, it is clear that coagulative tumour necrosis is a prognostic marker that can be readily applied in combination with more traditional variables (i.e., tumour size, TNM stage, and nuclear grade) (15) to enhance the performance of renal cell carcinoma scoring algorithms and predictive models currently in use to help assign follow-up and treatment in the clinical 
setting. The presence of coagulative necrosis in papillary renal cell carcinoma is of little prognostic significance (15). This finding is somewhat unexpected, given the high prevalence of tumour necrosis in papillary carcinoma and its association with other adverse pathological features. However, this finding highlights the fact that papillary renal carcinoma is relatively distinct from clear cell renal carcinoma. Papillary histologic type encompasses unique cytogenetic abnormalities, clinicopathological features, and prognoses, including a significantly more favourable cancer-specific survival in comparison to clear cell renal carcinoma (4).

Thus, coagulative tumour necrosis is a significant prognostic marker for clear cell and chromophobe renal carcinomas, and surgical pathologic evaluations should routinely record its presence or absence.

\section{Tumour necrosis in lung carcinoma}

Tumour necrosis has been reported as an indicator of poor prognosis in non-small cell lung carcinomas. The most commonly necrotic primary non-small cell lung cancer types are squamous cell and large cell undifferentiated carcinoma (2). Squamous cell carcinoma is a malignant epithelial tumour exhibiting keratinisation, pearl formation and/or intercellular bridges that originates from the bronchial epithelium. These features vary with degree of differentiation, being prominent in well-differentiated tumours and focal in poorly differentiated tumours. A central, comedo-type pattern of necrosis is typically observed in the higher-grade lesions (2). Primary pulmonary adenocarcinomas rarely demonstrate this alteration unless the tumours are extremely large or poorly differentiated. In a retrospective study, the prognostic implications of the extent of tumour necrosis were evaluated in non-small cell lung cancer and correlated with clinicopathological variables and the expression of Bcl-2, p53 and matrix metalloproteinase-9 (17). Tumour necrosis was graded as extensive or either limited or absent. Tumour necrosis correlated with T-stage, platelet count and p53 expression. No association was found with angiogenesis. On univariate and multivariate analysis tumour necrosis exhibited prognostic signifiance (17). These results indicate that extensive tumour necrosis reflects an aggressive neoplastic phenotype in non-small cell lung cancer and may improve the predictive power of the TNM staging system.

\section{Tumour necrosis in thyroid carcinoma}

Thyroid carcinomas of follicular cell origin are a spectrum of tumours ranging from the indolent, well-differentiated papillary carcinomas and minimally invasive follicular carcinomas to the almost universally lethal anaplastic carcinomas. In between these two extremes exist a group of tumours with an intermediate position at the histologic and prognostic levels. These neoplasms were termed 'poorly differentiated thyroid carcinomas' in their original description in the early 1980s $(3,13)$. The majority of authors concur on the existence of this entity; however, its histologic definition is subject to controversy $(14,16)$. For certain authors, these tumours are defined on the basis of a solid/trabecular or sclerotic 'scirrhous' growth pattern (13), whereas others suggest relying on 'histo- logical' grading (i.e., nuclear atypia, necrosis, and mitosis) irrespective of growth pattern and cell type (1). Hiltzik et al (6) have shown that poorly differentiated thyroid carcinomas defined on the basis of mitosis and necrosis constitutes a group of tumours that are more aggressive and more homogeneous than poorly differentiated thyroid carcinomas defined by growth pattern. Within this group of patients, microstaging (tumour size, the extent of capsular invasion, and extrathyroid extension), rather than growth pattern or cell type, is capable of stratifying patients into various prognostic categories (6). These findings indicate that grading of thyroid carcinoma based on increased mitotic activity, necrosis, nuclear pleomorphism and invasiveness is of high clinical and prognostic significance, whereas architectural grading has not yet been clearly proven to have any prognostic value $(6-9,11,18,19)$.

\section{Tumour necrosis in colorectal carcinoma}

Literature data regarding the prognostic significance of tumour necrosis in colorectal carcinoma are rare. However, the presence of tumour necrosis has been related to intra/-peritumoural inflammation and microsatellite status (5). Recently, Pollheimer et al (10) observed tumour necrosis in 365 (96\%) cases of colorectal adenocarcinomas, with 180 (47\%) tumours showing focal necrosis, 119 (31\%) moderate necrosis, and $66(17 \%)$ extensive necrosis. The extent of tumour necrosis was semiquantitatively assessed and recorded as absent, focal $(<10 \%$ of the tumour area), moderate $(10 \%-30 \%$ of the tumour area) or extensive ( $>30 \%$ of the tumour area). These authors demonstrated that the presence of 'extensive tumour necrosis' was an independent predictor of disease progression and cancer-specific survival in a model including a variety of established prognosticators, such as $\mathrm{T}$ and $\mathrm{N}$ classification, angioinvasion and tumour grade (10).

\section{Conclusion}

Tumour necrosis has garnered increased attention over the last few years, in part because a number of studies have now shown that tumour necrotic tissue is a significant prognostic marker with an independent effect on metastasis-free survival in patients with neoplasm. In particular, previous studies have indicated that surgical pathologic evaluation of tumour necrosis should routinely record its presence or absence. Such an assessment is easily performed through routine histological evaluation with a reasonably high rate of reproducibility among pathologists. To assess the value of tumour necrosis as a selection criterion for adjuvant treatment, future prospective trials should be conducted.

\section{References}

1. Akslen LA and LiVolsi VA: Poorly differentiated thyroid carcinoma - it is important. Am J Surg Pathol 24: 310-313, 2000.

2. Al-Nafussi AI and Hughes DE: Histological Diagnosis of Tumours by Pattern Analysis. Arnold, London, 1997.

3. Carcangiu ML, Zampi G and Rosai J: Poorly differentiated ('insular') thyroid carcinoma. A reinterpretation of Langhans' 'wuchernde Struma'. Am J Surg Pathol 8: 655-668, 1984.

4. Cheville JC, Lohse CM, Zincke H, Weaver AL and Blute ML: Comparisons of outcome and prognostic features among histologic subtypes of renal cell carcinoma. Am J Surg Pathol 27: 612-624, 2003 
5. Greenson JK, Bonner JD, Ben-Yzhak O, Cohen HI, et al: Phenotype of microsatellite unstable colorectal carcinomas: Well-differentiated and focally mucinous tumours and the absence of dirty necrosis correlate with microsatellite instability. Am J Surg Pathol 27: 563-570, 2003.

6. Hiltzik D, Carlson DL, Tuttle RM, Chuai S, et al: Poorly differentiated thyroid carcinomas defined on the basis of mitosis and necrosis: a clinicopathologic study of 58 patients. Cancer 106 1286-1295, 2006.

7. Lam JS, Shvarts O, Said JW, Pantuck AJ, et al: Clinicopathologic and molecular correlations of necrosis in the primary tumour of patients with renal cell carcinoma. Cancer 103: 2517-2525, 2005.

8. Leibovich BC, Blute ML, Cheville JC, Lohse CM, Frank I, Kwon ED, et al: Prediction of progression after radical nephrectomy for patients with clear cell renal cell carcinoma: a stratification tool for prospective clinical trials. Cancer 97: 1663-1671, 2003.

9. Moch H, Gasser T, Amin MB, Torhorst J, Sauter G and Mihatsch MJ: Prognostic utility of the recently recommended histologic classification and revised TNM staging system of renal cell carcinoma: a Swiss experience with 588 tumours. Cancer 89: 604-614, 2000.

10. Pollheimer MJ, Kornprat P, Lindtner RA, et al: Tumour necrosis is a new promising prognostic factor in colorectal cancer. Hum Pathol 41: 1749-57, 2010.

11. Rivera M, Ghossein RA, Schoder H, et al: Histopathologic characterization of radioactive iodine refractory FDG-PET positive thyroid carcinoma. Cancer 113: 48-56, 2008.
12. Roosen JU, Engel U, Jensen RH, Kvist E and Schou G: Renal cell carcinoma: prognostic factors. Br J Urol 74: 160-164, 1994.

13. Sakamoto A, Kasai N and Sugano H: Poorly differentiated carcinoma of the thyroid. A clinicopathologic entity for a high risk group of papillary and follicular carcinomas. Cancer 52: 1849-1855, 1983.

14. Sakamoto A: Definition of poorly differentiated carcinoma of the thyroid: The Japanese experience. Endocr Pathol 15: 307-311, 2004.

15. Sengupta S, Lohse CM, Leibovich BC, Frank I, et al: Histologic coagulative tumour necrosis as a prognostic indicator of renal cell carcinoma aggressiveness. Cancer 104: 511-520, 2005.

16. Sobrinho-Simoes M: Poorly differentiated thyroid carcinomas. Endocr Pathol 2: 99-102, 1996.

17. Swinson DE, Jones JL, Richardson D, et al: Tumour necrosis is an independent prognostic marker in non-small lung cancer: correlation with biological variables. Lung Cancer 37: 235-240, 2002.

18. Volante M, Landolfi S, Chiusa L, et al: Poorly differentiated thyroid carcinomas of the thyroid with trabecular, insular, and solid patterns. A clinicopathologic study of 183 patients. Cancer 100: 950-957, 2004.

19. Volante M, Collini P, Nikiforov YE, et al: Poorly differentiated thyroid carcinomas: The Turin proposal for the use of uniform diagnostic criteria and an algorithmic diagnostic approach. Am J Surg Pathol 31: 1256-1264, 2007. 